\title{
Von Literatur ist alles bis Emma Angeline. Ein Plädoyer für Booktube
}

From Literatur ist alles to Emma Angeline. A summation for Booktube

Jürgen Eder

\begin{abstract}
Booktube is a phenomenon that has existed internationally for some time and is drawing attention to itself through a constantly growing community as well as its originality and changeability. The article suggests integrating this variant of the digital literary public into studies of literature, not least because its effectiveness has been demonstrated above all in a younger reading public. The possibilities and limits of integration into literary practice are discussed, using selected examples from German and English-speaking countries. This is done on the basis of comparison with traditional literary criticism and the empirical description of what Booktube actually constitutes.
\end{abstract}

\section{Keywords}

Booktube, Literary Criticism, Literary Public, Literary Practice 
Sigrid Löffler hat im Jahre 2020 das Totenglöcklein der klassischen Literaturkritik geläutet, und begründet hat sie das u.a. damit, dass eine digitale Variante in Gestalt von AMAZON-Bewertungen an die Stelle klassisch-moderner Rezensionskunst getreten sei. ${ }^{1}$ Leser-Inkompetenz, Oberflächlichkeit und dergleichen sind die Urteile aus der Fachwelt zumeist gewesen, wenn es um diese nicht nur technisch neue Variante von Literaturbewertung ging. Aber nicht nur und erst durch ein Jahr „Distanz-Kultur“ in einer Pandemie haben sich die Diskurse und Kommunikation verändert, sondern schon einige Zeit vorher. Die „klassischen“ Sparten und Branchen des Feldes Literatur, also Literaturwissenschaft, Literaturkritik, Verlagswesen, zusammengefasst als „Literarische Öffentlichkeit“, haben darauf mehr oder weniger adäquat reagiert, diese Entwicklungen in ihre theoretische wie praktische Arbeit aufgenommen. Im Bereich Hyper-Texte, Digital Humanities, Autorschaft etc. wird länger schon über Konsequenzen für das Autor-Text-Problem diskutiert, von frühen Ansätzen bei Kittler² bis zu aktuellen etwa bei Zdeněk Pecka und seiner Habilitationsschrift zum Thema „Code und Kontext. Digitales Erzählen aus Österreich" . ${ }^{3}$ Hier haben sich im Bereich der Literaturwissenschaft wie der Literaturdidaktik schon integrative Ansätze etablieren können, auskristallisiert in den üblichen fachlichen Foren wie Publikationen, Forschungsprojekten und Konferenzen. $\mathrm{Zu}$ wenig bis kaum scheint mir dies freilich der Fall für die von Löffler explizit gemeinte dritte Säule des Literatur-Komplexes: den oder die Leser, für deren „Einbindung“ als Komplement zu Autor und Text nicht zuletzt die Literaturkritik zu sorgen hatte. Dabei steht auch für die neuen Varianten digitaler Leser-Reflexion das bekannte Rüstzeug der Rezeptionstheorie zur Verfügung, das sich auf Digital Reading ebenso applizieren ließe wie diverse Grundsätze praktischer Literaturkritik von Friedrich Schlegel bis zu Marcel Reich Ranicki.

Der folgende Beitrag möchte sich mit einem Phänomen befassen, das schon seit mehreren Jahren international eine Variante dessen zu sein scheint, was wir üblicherweise als „Literaturkritik“ bezeichnen. Dieses Format hat inzwischen eine quantitative wie qualitative Ausdifferenzierung erfahren, dass es mir legitim scheint, sich ihm auch als Literaturwissenschaftler ${ }^{4}$ zuzuwenden - nicht zuletzt unter der Perspektive der Einbindung in den Literaturunterricht: auch dann, wenn er vom Distanz- wieder in den Präsenzunterricht wechselt. Booktube als mediale Performance orientiert sich im Bereich der Vermittlung von Literatur, die uns als Hochschullehrer ebenfalls tangiert. Hier hat sich eine Form der Literatur-Diskussion herausgebildet, die nicht nur a) dem allgemeinen Trend zu den Digital Humanities folgt, sondern b) eine wachsende, interaktive Community umfasst, c) in vielem die Rolle von Literaturkritik übernimmt, d) gerade neue, jüngere Leser-Generationen anspricht und damit jene, die von der etablierten Kultur, Wissenschaft, Literatur als Leser oft schon aufgegeben wurden. Sowie e) als Booktuber nicht selten auch

1 Im Interview diese und weitere Aussagen zur Buch-Blogger-Szene: http://buzzaldrins.de/2014/02/20/ sigrid-loffler-im-gesprach/

2 Kittler, Friedrich: Aufschreibesysteme 1800/1900. München: Wilhelm Fink, 1985.

3 Auskunft des Verfassers.

4 Hier und im Folgenden wird das generische Maskulin verwendet. 
mit dem klassischen Literaturbetrieb verbunden sind: Studenten, Lehrer, Buchhändler, Verlage, selbst Literaturkritiker, die auch noch in den „alten“ Medien tätig sind.

Der Beitrag versteht sich deshalb als Anregung zur Diskussion aktueller Praxis im Literaturunterricht, sowohl in literaturwissenschaftlicher als auch literaturdidaktischer Hinsicht, was spezifisch für Deutsch als Fremdsprache relevant ist und bleibt. Er plädiert dafür, sich das Medium Booktube, seine Akteure, die Booktuber (oder unter welchen anderen Bezeichnungen sie noch auftreten mögen) als durchaus „wert“ vorstellen zu können, innerhalb von Lehre und Forschung eine Rolle zu spielen. Ich werde zunächst zu zeigen versuchen, dass die Praxis dieser Leser-Kanäle durchaus einige fundamentale Definitionsmerkmale klassischer Literaturkritik erfüllen. Sodann will ich ein kleines Glossar von Begriffen vorstellen, die fast überall anhängig sind und die Kommunikation der Booktuber sprachlich mitbestimmt. Anschließend werden einige ausgewählte Beispiele vorgestellt, mit ihren Spezifika, wobei die Auswahl sich auch an literaturwissenschaftlicher Anschlussmöglichkeit orientiert. Am Ende will ich dann versuchen, durch eine Gegenüberstellung von Schwächen und Stärken dieser digitalen Form von Literaturvermittlung abzuwägen, inwiefern sie für den praktischen Literaturunterricht empfehlenswert sein könnte.

Viele der Kriterien, die Wolfgang Albrecht ${ }^{5}$ in seiner Übersicht zur Literaturkritik als charakteristisch herausarbeitet, lassen sich durchaus auf Beiträge von Booktubern anwenden. Sowohl in der eher formal verfahrenden Beschreibung als auch in Fragen der Qualitätsbestimmung. Dass es keine wirklich normativen, allgemeinverbindlichen Maßstäbe gibt, ist keine originelle Erkenntnis, sondern Erfahrung recht eigentlich seit man spätestens ab der Romantik den „subjektiven Faktor“ zu einem entscheidenden Gradmesser gemacht hat. Der durchaus intendierte Versuch zur „Objektivität“, Nachvollziehbarkeit, Offenlegung der Kriterien ist doch ein eher hehrer denn realisierbarer Anspruch geblieben. Das gilt natürlich insbesondere, wenn es um eine „medial verbreitete öffentliche Auseinandersetzung mit vorwiegend aktueller Literaturproduktion [...] aus der Sparte >schöne Literatur< oder >Belletristik<" $<$ geht. Wer die diversen Runden des Literarischen Quartetts, auch im Nachfolge-Format von Thea Dorn und ihren Gästen, oder die relativ erfolgreiche Sendung von Denis Scheck Druckfrisch“, sieht und sich an Reich Ranickis Tiraden und Lobgesänge erinnert, die eine „normative Ästhetik“ gewiss nicht aufzuweisen hatte - der kann sich schwerlich über die „Subjektivität“, vorgeworfene „Beliebigkeit“ von Booktuber-Beiträgen beklagen. Hier wie dort ist erkennbar und gewollt Distanz zum akademischen Umgang mit Büchern, geht es um - mehr oder weniger eingestandene - personale Wertungen, Haltungen, Urteile. Dies konzediert, wird man Unterschiede eher in rhetorischen Fähigkeiten oder performativer Professionalität ausmachen können. Dass hier bei einigen Booktubern, v.a. aus dem angloamerikanischen Raum, ein Grad erreicht ist, der auch die Namhaftesten der traditionellen Literaturkritik buchstäblich „alt“ aussehen lässt, wird in den später folgenden Einzeldarstellungen angesprochen. Zunächst soll, an Albrechts Überblick orientiert, gezeigt

5 Albrecht, Wolfgang: Literaturkritik. Stuttgart/Weimar: Verlag J.B. Metzler, 2001.

6 Ebd., S.1. 
werden, wo Booktuber Literaturkritik im eher bekannten Sinne ohnehin schon recht nahestehen.

Literaturkritik wie Booktube sind „integraler Bestandteil des Öffentlichkeitsbereiches, für den die Bezeichnung >literarische Öffentlichkeit< geläufig ist."7 Dass diese spezifische Öffentlichkeit und demnach auch ihre Institutionen, Vermittlungsformen in einer lange währenden Legitimationskrise stecken, muss kaum beglaubigt werden. Ein Weg, sich damit auseinanderzusetzen, darauf zu reagieren, ist es, neue Formate, sowohl technischer als auch inhaltlicher, performativer Art zu suchen und anzubieten. Für längere Zeit bestimmte „Literatur im Fernsehen“ diese Funktion, doch inzwischen ist auch dort die Marginalisierung so weit fortgeschritten, dass neue, unabhängigere, multimedial agierende Kanäle diese Aufgabe übernommen haben. Eigentlich finde ich es erstaunlich und es ist wohl ein weiteres Indiz für die relative Schwerfälligkeit des „Tankers“ Öffentlich-Rechtliches Fernsehen, dass solche durchaus, v.a. bei jüngeren Leserinnen und Lesern, erfolgreiche Plattformen wie Poetry Slam oder eben Booktube kein Interesse gewidmet wird. Hier wiederholt sich offenbar die relative Sprachlosigkeit zwischen politischem und medialem Establishment und einer digitalen Jugendbewegung, wie sie von anderen Beispielen her bekannt ist. ${ }^{8}$ Längst gibt es eine „Mehrzahl literarischer Öffentlichkeiten“9, die freilich noch zu sehr hierarchisiert werden, blickt man auf die großen Sender, egal ob privat oder staatlich, und den Booktubern und anderen, deren „Produktionsmittel“ zwar hinreichen, um ihr Format in die Öffentlichkeit zu stellen, aber natürlich nicht in vergleichbarem Umfang. Man könnte Booktube im Bereich der Literaturkritik vielleicht eine „Graswurzel-Revolution“ nennen.

Der Prozess, in dem „der Kritiker erst selber zum Leser, dann zum Autor wird“10, ist beiden Arten Literatur zu rezipieren und weiterzuvermitteln gemeinsam. Freilich wird man in unseren Tagen schwerlich noch von „priviligiertem“ Leser sprechen können, dessen „Rezeptionshaltung von überindividueller Bedeutung ist"11 - der Radius der erreichten Adressaten ist überschaubar, dürfte sich rein quantitativ allerdings inzwischen in Richtung der Booktuber-Zahlen verschoben haben. Wie bei den traditionellen Literaturkritikern haben die Verlage inzwischen auch der Booktuber-Community ihre Aufmerksamkeit geschenkt. Der Markt scheint hier einmal mehr wacher als die öffentlich-rechtliche Medienlandschaft. Einige Verlage, beispielsweise Luchterhand, haben bereits eigene Book-Channels etabliert, die anderen versenden angefragt oder ungefragt Rezensionsexemplare an Booktuber. Diese Verbindung machen die bei nahezu allen hier gesichteten Beispielen eingeblendeten Hinweise wie „Enthält unbezahlte Werbung“ deutlich. Sind die Kanalbetreiber bekannter, überschreiten eine gewisse Grenze der Abonnenten-Zahl,

7 Ebd., S.5.

8 So die vielbeachtete Auseindersetztung des youtubers Rezo mit der CDU und deren Reaktionen. Das Video Rezos unter https://www.youtube.com/watch?v=4Y11ZQsyuSQ. Artikel der Süddeutschen Zeitung über Reaktionen der CDU: https://www.sueddeutsche.de/politik/nach-fall-rezo-ziemiak-will-newsroom-einfuehrenund-arbeitsablaeufe-in-cdu-zentrale-aendern-1.4622154

9 ALBRECHT, s. Anm. 4, S.7.

10 Ebd., S.9.

11 Zitiert ebd. 
werden die Verlage zumeist von sich aus aktiv, ansonsten bitten Booktuber manchmal selbst um Rezensionsexemplare. Der „durchschnittliche“ Booktuber allerdings erwirbt die Bücher selbst. Dazu muss gesagt werden, dass ein kleiner Kanal natürlich eher auf Entgegenkommen angewiesen ist als Kritiker in größeren Medienunternehmen und man deshalb vermuten könnte, sie seien auch positiver mit Urteilen zu überlassenen Büchern. Insgesamt habe ich aber diesen Eindruck nicht. Zumal, wie gesagt, das Gros die Bücher selbst oder auf anderen Wegen erwirbt.

Ein Trend, den Albrecht 2001 diagnostiziert, dass es nämlich einen wachsenden Anteil an Frauen unter Literaturkritikern gibt, ist für die Booktuber-Gemeinde noch weit zutreffender. Hier bildet sich durch eine deutlich weibliche Majorität auch die Tatsache $\mathrm{ab}$, dass die Leserschaft für Belletristik weitestgehend weiblich ist. Das Spezifikum bei den Booktuberinnen im Vergleich mit ihren weiblichen Pendants in der professionellen Branche liegt darin, dass sie fast durchgehend jünger sind, die meisten zwischen 20 und 30. Hier wird keine vorgezeichnete Laufbahn absolviert, im Prinzip genügen einige basale Eigenschaften psychologischer (Selbstbewusstsein, Präsentations-Geschick, Selbstinszenierung) und technischer Natur (Equipment).

Albrechts 2001 wohl noch mit skeptischem Unterton gestellte Frage, ob das Internet der Literaturkritik eine mehr als nur kurzfristige neue Seite hinzugewinnen wird: „ob die Publikumsresonanz auf die [...] Angebote im Internet den Anfangsreiz eines neuen Mediums überdauert"12 - diese Frage ist inzwischen mit einem eindeutigen „Ja“ zu beantworten.

Ein Vorteil der Booktuber-Vermittlung von Literatur scheint mir auch zu sein, dass die Skala der dort besprochenen Bücher - je nach Interessenlage des Kanals und seines Betreibers - vielfältiger ist als in der klassischen Literaturkritik. Dort gibt es für alle Feuilleton-Redaktionen die so gut wie deckungsgleiche Notwendigkeit, den „neuen Handke, die neue Zeh" usw. zu besprechen. Dies gibt es in dieser Form bei Booktubern nicht, und gelegentlich übernehmen sie die wichtige Aufgabe, Schriftstellerinnen und Schriftsteller in den Blick zu bekommen, die sonst nicht wahrgenommen werden. Die Fülle der Kanäle erhöht die Wahrscheinlichkeit, dass für jeden Rezipienten seine favorisierten Genres auch verhandelt werden. Die Kriterien der Auswahl bieten deshalb insgesamt doch wesentlich „mehr als ausschnitthafte Überblicke“ ${ }^{13}$

Ein essentieller Faktor ist beim traditionellen Literaturkritiker wie beim Booktuber die „Subjektivität bzw. Individualität des Kritikers“. ${ }^{14}$ Im gegenwärtigen System von Literaturvermittlung wird man ohne Elemente von Unterhaltung oder Personality Image nicht auskommen, mag man das kulturkritisch beklagen oder auch nicht. Eine Generation junger Digital Natives, die sich in diversen Apps und Varianten optischer Selbst-Repräsentation vom Selfie bis zu zum youtube-Video in dieser Kunst geübt haben, hat das nötige Know How längst entwickelt und bedient sich dieser Fertigkeiten teilweise virtuos und beeindruckend. Die den Profis oft abgesprochene Leser-Nähe wird hier unmittelbar

12 Ebd., S.27.

13 Ebd., S.34.

14 Ebd., S.69. 
optisch und akustisch verifizierbar, mag es auch gelegentlich durchschaubar inszeniert wirken.

Auch Andreas Reckwitz beurteilt die Entwicklung eher kritisch, sieht im Hyper Reading eine „neue dominante kulturelle Praxis“, ${ }^{15}$ dem gegenüber Deep Reading „eine neue gegenkulturelle Praxis“16 besserer Art entstanden sei. Die von Reckwitz hier angenommene „Ausdünnung“ und „Veralltäglichung“17 des Lesens, wie sie durch die diversen digitalen Revolutionen erfolgt sei, sehe ich zwar in gewissen Aspekten der Booktuber-Kultur durchaus, allerdings keineswegs in dieser Pauschalität. Im Gegenteil: Viele dieser Kanäle scheinen mir Anhänger, Verfechter eher des Deep Reading, wenngleich natürlich das Medium auf Abwechslung und Originalität hin orientiert ist. Die Gratwanderung gelingt einigen Booktubern jedenfalls besser als von vielen befürchtet. Es kommt dann zu einer Kreuzung aus Hyper Reading mit Deep Reading, wo in den Zwischenräumen interessante Hybride entstehen, die in der traditionellen Literaturkritik nicht zu finden wären. Dazu kommt die Vernetzung verschiedener digitaler Formen wie Instagram, Facebook und youtube, ein Multitasking, das nicht notwendig Oberflächlichkeit, „Zerstreutheit“ bedeuten muss und auf jeden Fall eine Erweiterung des Kreises bringt, der über Literatur spricht - „Sprechen“ in verschiedenstem Sinne.

Reckwitz ist hingegen zuzustimmen, dass die Geschichte der Moderne „regelmäßig Gegenkulturen"18 herausgebildet hat, wenn sich Genres und Formen als nicht mehr hinreichend vermittelbar erwiesen. Dies gilt auch für die Funktion der Literaturkritik. Die zu beobachtende Überlagerung alter Strukturen „mit den neuen“, wobei die alten „als teilweise recht lebendige Relikte vorhanden"19 bleiben, ist ein Prozess, der sich in der Entfaltung einer Moderne immer wieder beobachten lässt. Und so wird mancher Rezipient von Booktube eher traditioneller Couleur überrascht sein, wie viele „Relikte“ dort noch zu finden sind. Es ist dazu aber notwendig, nicht a priori sich am digitalen, also technisch-performativen Begleitcharakter des Diskurses über Bücher und ihre Hintergründe zu stören. Auch nicht an der klaren Ausrichtung auf die personale Referenz, die durch starke optische wie lebensweltliche Präsenz in diesen Beiträgen deutlich wird. Vor allem finde ich etwas zu simpel, den Vorwurf pauschal auf die junge Generation zu münzen, wie das etwa auch bei Wolf Singer geschieht: „Vor allem junge Menschen erleben diese totale Vereinnahmung als prickelnden Reiz und erliegen der Verführung“20 digitaler Berieselung. Wer sich durch eine Reihe von Booktube-Kanälen sieht und hört, wird dies allzu rasche Urteil nicht teilen können, eher Wallensteins Urteil über die Jugend umkehren wollen $\mathrm{zu}$ „Schnell fertig ist das Alter mit dem Wort“. ${ }^{21}$ Natürlich gibt es auch rasche, manchmal hektische Schnittfolgen, die Unruhe permanenter Bildwechsel.

15 Reckwitz, Andreas: Kleine Genealogie des Lesens. In: (Hrsg.): Raabe, Katharina und Wegner, Frank: Warum lesen. Mindestens 24 Gründe. Frankfurt/M.: Suhrkamp Verlag, 2020, S. 33.

16 Ebd.

17 Ebd.

18 Ebd., S.42.

19 Ebd., S.43.

20 Singer, Wolf: Immaterielle Realitäten. In: s. Anm. 14, S. 160.

21 Schiller, Friedrich: Wallensteins Tod. III/2. In: (Hrsg.): Fricke, Gerhard und Göpfert, Herbert G.: Friedrich 
Aber die Aufforderung, das Angebot, eine gewisse Zeit lang, zumeist zwischen 20 und 45 Minuten, jemandem zuzusehen bzw. zuzuhören, der oder die über Bücher spricht und dabei auch noch einen kleinen Einblick in eine Leser-Welt gibt - vom Bucherwerb über Markierungstechniken beim Lesen bis zur Erstellung einer Regalordnung oder Tipps zum Führen eines Book-Journals - der wird solche Stereotype kaum aufrechterhalten können.

Einen letzten Aspekt in diesem allgemeinen Vergleich von klassischer Literaturkritik und Booktube, der bereits angedeutet wurde, möchte ich noch einmal aufgreifen, einer Aufforderung Terry Eagletons folgend: „Würden wir uns von Literatur nur dann inspirieren lassen, wenn sie unsere eigenen Vorlieben und Interessen spiegelte, wäre das Lesen eine Art Narzissmus". ${ }^{22}$ Solcher Narzissmus scheint mir in der traditionellen Literaturkritik nicht unüblich. Einerseits pflegt man da seine Sympathien und Antipathien, wie im Falle Reich Ranickis beispielweise gegenüber Philip Roth und Martin Walser. Andere fühlen sich häufig als verkannte Literaturwissenschaftler und schielen auf den Beifall von dieser Seite, indem sie nur allzu bereitwillig zu „Großem“ und „Kompliziertem" greifen, und das gerne auch stilistisch. Welch ein Angebot verschiedenster Genres und Stillagen hingegen bei den Booktubern! Mag man beckmesserisch manches davon als nicht „literaturaffin“ verwerfen - es bleibt immer eine reiche Fülle von Titeln, Reihen, Präsentationsweisen, auf die man im Betrieb der Literaturkritik selten genug stößt. Neugierde, Neigung, Subjektivität mögen nicht immer die besten Ratgeber im Prozess der Urteilskraft sein - fehlen sie aber gänzlich, so wirken Verrisse wie Hymnen eher wie das Papier, auf dem sie gedruckt sind.

Bevor ich zu einigen wichtigen Termini der Booktuber-Szene komme, möchte ich das „Projekt“ Booktube zumindest ansatzweise allgemein charakterisieren. Dass er aus den Begriffen Book für Buch und Tube für Röhre (als Kanal, Sender, man mag an Radio- oder Fernsehröhren denken) gebildet wurde, ist evident, in der Bezeichnung ist natürlich auch der Zusammenhang mit youtube gegeben. Wer exakt als Gründer von Booktube zu gelten hat, ist ebenso unbekannt wie ein genaues Gründungsdatum zu nennen. Man erwähnt häufig das Jahr 2010, seitdem sich die Community langsam und wachsend aufbaut. Allerdings gibt es ähnliche Ansätze auch schon vorher. Das Wachstum seitdem aber darf als exponentiell bezeichnet werden, es gibt Hunderte, eher wohl Tausende Kanäle weltweit, mit jeweils Tausenden, manchmal sogar über Hunderttausenden Abonnenten, von den Aufrufen gar nicht zu sprechen. Booktube-Kanäle werden in allen Weltsprachen betrieben, obgleich Englisch überwiegt. Das Spektrum der Genres ist schier unbegrenzt, reicht auch in die nicht-fiktionale Literatur. Für die Intention dieses Beitrags beschränke ich mich weitgehend auf Formate, die sich auch mit „Klassikern" und verschiedenen Varianten von Gegenwartsliteratur beschäftigen, wie sie auch in Vorlesungen oder Seminaren vorkommen können. Ein englischsprachiger Beitrag für die Deutsche Welle spricht davon, dass Booktuber den Buchmarkt verändern würden:

Schiller. Sämtliche Werke. Zweiter Band. Dramen II, München 1991: Hanser Verlag, S.434. Originalzitat lautet: "Schnell fertig ist die Jugend mit dem Wort".

22 Eagleton, Terry: Literatur lesen. Eine Einladung, Stuttgart 2016: Reclam, S.221. 
„BookTubers can definitely boost book sales“23. Die Verlage, v.a. im englischsprachigen Raum - wo die Abonnentenzahlen der Tuber zumeist um ein Vielfaches höher liegen als bei ihren deutschen Pendants - haben diese Markterweiterung längst erkannt und arbeiten gerne mit ihnen zusammen. Sponsoring ist ein Faktor auch hier, v.a. in Deutschland aber beschränkt sich dies in der Regel (noch?) auf Überlassung von BesprechungsExemplaren. Booktuber verfügen, und hier geht es natürlich weit über bekannte Formen von Literaturkritik hinaus, sozusagen über ihre eigenen Produktionsmittel, in Gestalt „mehrdimensionaler“ bzw. multimedialer Mittel, um ihre Bücher an die Frau oder den Mann zu bringen.

Sie arbeiten mit filmischen Schnitttechniken, oft schon professionellen Kameras und weiterem Equipment. Zumindest im jeweiligen Intro wird zumeist Musik, eine Art Jingle, eingespielt, als Wiedererkennungseffekt - findet sich aber nicht selten auch in anderen Partien des Videos. Während in den USA ein Booktuber-Kanal schon bis zu 5 Mitwirkende hinter der Kamera haben kann, ist es in deutschen Videos in der Regel eine Einzelperson. Diese bestimmt dann auch das Setting, und das reicht vom jeweiligen Ambiente, in dem gefilmt wird bis zur Kleidung. Hier gibt es wieder sichtbare Unterschiede: Während die für diesen Beitrag ausgewählten deutschen Booktuber in der Regel vor ihren Bücherregalen posieren, findet dies z.B. bei Emma Angeline aus London zumeist in ihrem Schlafzimmer, gelegentlich auch auf ihrem Bett statt. Es soll so „ungezwungen“ wirken, scheint mir aber, wie auch nicht selten auffällige Kleidung, auch sexualisierte, auf Generierung von Klicks zu zielen, die nicht nur literarisch motiviert sind. Ansätze dazu gibt es, bereits zunehmend, auch beim deutschen Format, aber definitiv noch als Ausnahme. Natürlich ist, wie bei Buchkritik, die optisch vermittelt, etwa bei Literatur im Fernsehen, dem „Literaturclub“ des Schweizer Fernsehens oder in der „Next Generation“ des Literarischen Quartetts um Thea Dorn Personality ebenfalls ein Schlüsselmoment. Sie besteht aus Intellektualität, Kompetenz, selbstverständlich - aber eben auch aus „Optik“. Die Vermarktung sowie Aspekte der Werbung haben sich längst in der Buchbranche etabliert, und es wäre heuchlerisch, dies in Abrede zu stellen oder nur Booktube anzulasten. Wichtig ist natürlich bei diesem Medium die Stimme - nicht nur ihre Tonlage, sondern auch ihre Fähigkeit, über einen gewissen Zeitraum hin zu tragen. Natürlich kann schnitttechnisch nachgebessert werden, allerdings fällt dies bei Häufung unangenehm auf.

Über die Psychologie der Booktuber ließe sich eine eigene Studie schreiben. Natürlich vermutet man eine gewisse Selbstsicherheit, fast bis zum performativen Exhibitionismus gehend. In den diversen Selbst-Auskünften, die immer wieder in Form von Vlogs oder Tags eingeschaltet werden, wird dann aber oft das Gegenteil bekannt, von typischen „Nerd“-Merkmalen, Zurückgezogenheit und Unsicherheit berichtet. Diese Home Stories lassen, natürlich unter kritischem Vorbehalt gegenüber allzu betonter „Authentizität“, doch den Schluss zu, dass sich die Macher dieser Kanäle nicht gerade zur Feier-Jugend rechnen.

23 Zit. im Beitrag der Deutschen Welle: https://www.dw.com/en/how-booktubers-are-changing-book-marketing/a-18633901 
Dass unter ihnen junge Frauen überwiegen, jüngere Menschen generell, wurde schon gesagt: Es gibt Einzelne, die schon im Alter von 16 begonnen haben, einen Booktube-Kanal zu betreiben. Sozial gesehen handelt es sich zumeist um Studenten, Lehrer, ehemalige oder noch beschäftigte Buchhändler, v.a. im deutschen Bereich. Ein Kritiker wie Markus Gasser, der auch professioneller Literaturkritiker der NZZ ist, bildet eine Ausnahme - aber die Herangehensweise ist bei ihm ähnlich wie bei den anderen Booktubern.

Ein ganz wesentlicher Aspekt sei hier last but not least vermerkt: Die Kommentarfunktion der Kanäle, die Verlinkung mit weiteren Medien ihrer Betreiber bei Instagram, Twitter, Facebook - aber auch mit anderen Booktubern. Die immer wieder vorgebrachte Aufforderung diese Vernetzung zu nutzen, v.a. aber auch Kommentare zu hinterlassen, machen die Intention deutlich, eine möglichst intensive Kommunikation zu schaffen. Nicht alle Booktuber reagieren auf die Kommentare, aber die für diesen Beitrag ausgewählten versuchen durchaus, sich nicht mit Likes oder Abos zu begnügen. Die Frequenz der Kommentare richtet sich zumeist proportional an der Anzahl der Abonnenten und Aufrufe aus. Nicht selten kommt es dort zu kenntnisreichen und engagierten Diskussionen über Bücher, Urteile, Empfehlungen.

Zuletzt sei in dieser allgemeinen Charakterisierung noch auf eine Tendenz hingewiesen, die ich bedauerlich finde und die mittelfristig die unweigerlichen Stärken des Projekts Booktube untergraben würde. Einige, die Zahl wächst, vermischen das Hauptthema Bücher immer häufiger mit anderem, das sich unter dem Begriff Lifestyle zusammenfassen lässt. Kosmetik, Kleidung, Ernährung usw., auch politische Statements, sie scheinen mir nicht recht ins originäre Konzept zu passen. Ersichtlich geht es darum, mehr potentielle Seher, v.a. Abonnenten zu generieren. Es wird wohl davon abhängen, wie viele Nutzer primär oder ausschließlich am Thema Literatur interessiert sind und sich dann vermutlich irgendwann gegen eine solche Entwicklung wenden. Oder ob diese Hybridität, man darf schon sagen: Kommerzialisierung, in Kauf genommen wird.

Die Booktuber-Community hat, wie andere Gruppen im Rahmen von Social Media, eine eigene Terminologie entwickelt. Sie besteht weniger aus spezifisch literaturtheoretischen oder literaturkritischen als vielmehr performativen Begriffen, die zur Kommunikation zwischen Booktubern und Nutzern geprägt wurde. Sie ist größtenteils von englischen Begriffsbildungen abgeleitet, zumeist mehr oder weniger geglückt einfach übersetzt. Das Repertoire erreicht selbstverständlich nicht die Dichte literaturwissenschaftlicher Fachsprache, hat sich aber über die Jahre stetig weiterentwickelt und ist nach wie vor in Gang. Deshalb ist die folgende kleine Übersicht repräsentativ nur in dem Sinne, als dass sie häufige Begriffe und Bezeichnungen aufführt. Dabei verfahre ich im Wesentlichen deskriptiv.

SUB - Stapel ungelesener Bücher (Engl. vgl. WTR - Want to Read). Darunter versteht man eine wechselnde Anzahl von Büchern, die noch nicht gelesen aber - per „Stapel“ - zur Lektüre in absehbarer Zeit vorgesehen sind. Dabei handelt es sich um Neuerwerbungen oder um Bücher aus einem Bestand, die man „längst schon“ lesen wollte. So ein Stapel ist zahlenmäßig nach oben prinzipiell offen, bewegt sich aber zumeist um die 10 - 20 Exemplare. Die Kriterien dafür können sogenannte Reading Challenges sein 
oder auch einfach nur der Wunsch, ein bestimmtes Kontingent, manchmal eher „sportiv“, zu lesen.

LESERUNDEN - (Engl. Bookcircle). Eine digital-virtuelle Variante des Literarischen Quartetts wenn man so will, bei dem sich mehrere Booktuber zusammenfinden, das gleiche Buch lesen und sich darüber via youtube oder auch Facebook, Instagram oder WhatsApp austauschen.

BOOK-CHALLENGE (Deutsch vielleicht: Lese-Herausforderung). Es handelt sich um selbst gestellte Aufgaben oder durch sogenannte Tags von anderen Tubern formulierte Herausforderungen, eine gewisse Menge an Büchern etwa eines bestimmten Genres zu lesen. Die Variationsbreite ist dabei recht groß, kann zeitlich (z.B. 7 Tage) oder auch durch Anzahl der zu lesenden Bücher (auch hier oft 7) limitiert sein. Diese Reading Challenges sind oft sehr kreativ, suchen den Spaß, auch das Experiment, unorthodoxe Lektürekriterien. Das kann der Umfang sein (ein Buch mit über 500 Seiten), ein Buch, das verfilmt wurde, ein Buch mit einer Zahl im Titel oder ein Buch, das ein Freund empfohlen hat usw. Wenn es sich um eine Jahres-Challenge handelt, sind es dann am Ende meist 20-25 solcher ausgewählten Bücher. Der Reiz ist evident: Man soll sich über die üblichen Kriterien hinauswagen, auch dem Faktor Zufall bei der Lektüre eine Chance geben. Diese spezifische Challenge wird oft von anderen Booktubern aufgegriffen, um dann Erfahrungen, Erfolge oder Misserfolge damit in einem eigenen Video zu kommunizieren. Auf das dann wieder reagiert wird, in Form anderer Aufgaben oder als Reaktion auf die gemachten Erfahrungen. So kommt ein anhaltender, abwechslungsreicher Diskurs innerhalb der Community in Gang - den man sich für den akademischen Betrieb nur wünschen kann.

LESEMONAT - Etwas Ähnliches ist der Lesemonat, der retrospektiv angelegt ist: man blickt zurück, was man im vergangenen Monat gelesen hat, in Form von kürzeren Besprechungen. Je nach Ambitionen liegen die Zahlen da zwischen 3 und 15 Büchern, in Zeiten des Lockdowns sind sie natürlich tendenziell höher. Ein Jahresrückblick bietet analog dazu, zumeist im ersten oder zweiten Monat des folgenden Jahres, einen Rückblick auf ein Lesejahr, häufig in Gestalt einer Wertungs-Liste mit Erklärungen, wie diese Skala zustande kam. Hier reicht die Facette von relativ einfachen Kriterien wie „Hat mir gefallen/super gefallen“, „fünf Sterne“ - bis zu differenzierten, jeder Fachkritik durchaus gewachsenen Argumentation. Wie generell hängt es wieder davon ab, welche Herangehensweise und nicht zuletzt welches Publikum der jeweilige Booktuber im Auge hat.

Ein Format, das die Funktion einer Art von „Appetizer" hat, ist das Öffnen von bestellten, eingehenden Buchpaketen, dem sog. UNPACKING. Hier wird der Zuseher in die Vorfreude der ersten Kontaktnahme mit neuen (gelegentlich auch gebraucht bestellten) Büchern einbezogen. Die ausgepackten Bücher werden kurz vorgestellt, häufig durch Auszüge aus den Klappentexten, verbunden mit den eigenen Erwartungen. Zumeist wird dann noch auf ein späteres Video hingewiesen, in dem diese Bücher ausführlicher besprochen werden sollen.

Die BÜCHERREGAL-TOUR (Engl. Bookshelf Tour) erscheint gleichfalls bei nahezu allen Booktubern. Sie tastet Regale mit der Kamera ab, nimmt manchmal ein Buch heraus und spricht kurz darüber. Da die meisten Booktuber über eine ansehnliche kleine 
Bibliothek verfügen, wird diese Tour meist in mehreren Folgen produziert. Dies bietet dem Interessenten auch eine Vorstellung davon, „wes Geistes Kind“ der jeweilige Booktuber ist, wo seine Interessen, Neigungen liegen und was demzufolge für Besprechungen zu erwarten sind. Häufig sind diese Regal-Fahrten mit persönlichen Geschichten zu einzelnen Büchern verbunden, was dem Anspruch von persönlichem Bezug, Intimität entgegenkommt, das durch das Setting (private Räume, Stimmungen etc.) noch weiter beglaubigt wird. Diese Bücherregal-Tour ist im Prinzip eine Metamorphose der berühmt-berüchtigten Frage: „Haben Sie das alles gelesen?“, über die beispielsweise Klaus Walther ein amüsantes Kapitel in seinem Bändchen „Bücher sammeln“ geschrieben hat $^{24}$ - oder auch Annie Ernaux, die erzählt, dass sie beim Anblick ihrer Bibliothek sofort mit einem „Universitätsabschluss“ in Verbindung gebracht wird..$^{25}$ "Sage mir, was in deinen Regalen steht, und ich sage dir, wer du bist“... so einfach ist es natürlich nicht, aber ein wenig hat es davon doch auch.

REZENSIONEN, die von diversen Kanälen eingebaut werden, nähern sich am weitesten der klassischen Rezension. Sie werden in der Regel von Booktubern gemacht, die überdurchschnittlich oft nicht nur Neuerscheinungen, sondern auch Texte des literarischen Kanons besprechen. Als neueres Beispiel kann man hier auf KainundAbel und seine Thomas-Mann-Besprechungen hinweisen ${ }^{26}$ oder Leonieoverthere mit ihrer Würdigung von Max Frischs Montauk. ${ }^{27}$ Dabei wird einzelnen Büchern wesentlich mehr Zeit eingeräumt als üblicherweise - denn die durchschnittliche Zeit für eine Buchvorstellung bewegt sich zumeist im Intervall von 2-5 Minuten, abhängig von der absoluten Dauer des Beitrags, der sich normalerweise zwischen 15 und 20 Minuten einpendelt.

Im Folgenden möchte ich einige ausgewählte Kanäle vorstellen, 5 deutschsprachige und 2 englischsprachige. Kriterien für die Auswahl waren Qualität und Nähe zum Aspekt Literaturkritik - etwa durch die Tatsache, dass regelmäßig auch über Klassiker gesprochen wird. Repräsentativität war ein weiteres Kriterium: Geschlecht, Alter, Milieu. Neben dem qualitativen gibt es auch einen quantitativen Aspekt: Der Kanal sollte mit einer gewissen Regelmäßigkeit aktiv sein. Andererseits habe ich mich nicht an der bloßen Abonnentenzahl oder Likes und Aufrufen orientiert. Schließlich: Es sollte der Kanal eindeutig Bücher und Bücher-Welten zum Inhalt haben. Ich werde die ausgewählten Booktuber zunächst mit einigen Angaben empirischer Art vorstellen und dann eine Charakterisierung und Wertung des Formats versuchen.

Ich beginne mit Literatur ist alles, betrieben von Markus Gasser, nach der Selbstbeschreibung in seinem Kanalinfo „Schriftsteller und Dozent für Neuere Deutsche und allgemeine Literaturwissenschaft“, der in Zürich lebt und eine „Plattform in der Welt der neuen Medien bieten“ will, um seine Liebe zur Literatur mit „einem begeisterungsfähigen Publikum zu teilen“. ${ }^{28}$ Er betreibt Literatur ist alles seit 2017, verfügt über aktuell

24 Walther, Klaus: „Haben Sie das alles gelesen?“ In: Ders. Bücher sammeln, München 2004: dtv, S. 17-23.

25 ERNAUX, Annie: Trennen, Verbinden. In: s. Anm. 14, S. 79.

26 https://www.youtube.com/watch?v=q7JFbXlekZA

27 https://www.youtube.com/watch?v=LT46gQvzP2A

28 https://www.youtube.com/c/LITERATURISTALLES/about 
13.300 Abonnenten und bespricht primär Werke der klassischen Moderne wie Thomas Mann, Dürrenmatt, Musil u.v.a., aber auch Texte der Popkultur wie Douglas „Per Anhalter durch die Galaxis“ oder Stephen King. Gassers Kanal ist sicherlich derjenige, der etablierter Literaturkritik am nächsten kommt. Neben engagierten Besprechungen der Welt- und Gegenwartsliteratur stehen Specials, Themenvideos, die dann den gewohnten zeitlichen Rahmen sprengen. Sie können aktuell sein (z.B. „Revolte gegen das Coronavirus“" ${ }^{29}$ oder in einem „10.000 Subscriber Special“ mit einem anderthalbstündigen Beitrag die Frage erörtern: „Was ist große Literatur?“. ${ }^{30}$ Gassers Video-Archiv ist beinahe schon eine Welt-Literatur-Geschichte mit „Seitengebäuden“ zu verschiedenen neueren Büchern und Genres. Seine Kenntnis der Weltliteratur ist für viele sicher einschüchternd und vermutlich findet sich unter seinen Abonnenten eine erhebliche Anzahl „Fachpublikum“, also Literaturwissenschaftler, Studenten, Menschen aus dem „Literaturbetrieb“. Die Grenze zwischen Seminar und Blog ist fließend, allerdings häufig in Richtung des ersteren verschoben. Dazu trägt auch die performative Manier Gassers bei: Er hat, bei aller Leidenschaft, doch etwas Belehrendes, Dozierendes, man fühlt sich an einen audiovisuellen Hörsaal erinnert. Insgesamt aber kann man gewiss sagen, dass sein Kanal von allen hier besprochenen Anforderungen von fachwissenschaftlicher Seite am nächsten kommt. Gasser verzichtet weitestgehend auf Einblicke ins private Umfeld, die „persönliche“ Ebene beschränkt sich auf imposante Rundgänge in seiner Heim-Bibliothek, die dann auch wieder etwas von Überwältigung hat.

Hinter dem mysteriösen Kanalnamen KainundAbel steht ein „freundlicher Bibelexeget“ namens Max, wohnhaft in Leipzig, der „vor allem Klassiker und weltweite Gegenwartsliteratur“ liest und analysiert und sich an „enthusiastische Liebhaber guter Literatur"31 wendet. Er ist Lehrer an einem Gymnasium und bei Booktube seit 2010, womit er in Deutschland zu den Pionieren gehört. Sein Kanal hat aktuell 2410 Abonnenten, für deutsche Verhältnisse durchaus ansehnlich. KainundAbel lädt so gut wie jede Woche zumindest ein Video hoch und behandelt dabei alle Booktube-Formen. Von gelesenen Büchern eines Monats, dem Jahresrückblick über Tags wie „Meine Lieblingsautoren“32 oder einer Erklärung, wie er zu Booktube kam und was für ihn diese Plattform bedeutet, reicht die Skala. Schwerpunktthemen finden sich ebenfalls, etwa „Triviales - Eine Tirade in sechs Kapiteln“33 oder auch, korrespondierend eigenen Erfahrungen im Beruf „Bücher und Schule“. ${ }^{34}$ Im Mittelpunkt steht aber die Besprechung und Deutung von Klassikern, auch sonst eher ungewöhnlichen Beispielen wie Arno Schmidt. Eine Besonderheit des Kanals ist die Originalität der Video-Titel, die Lust am Sprachspiel und Zitat verrät. Wie im Falle Gassers hat sich hier eine regelrechte Video-Bibliothek von Besprechungen über 11 Jahre angesammelt, verwendbar durchaus im Literaturunterricht nicht nur der

29 https://www.youtube.com/watch?v=qcAugg_kHY0

30 https://www.youtube.com/watch?v=9Zqg0glOhHI

31 https://www.youtube.com/user/KainUndAbel2/about

32 https://www.youtube.com/watch?v=8eca-Es9Dkk

33 https://www.youtube.com/watch?v=omHRyX2ErOg

34 https://www.youtube.com/watch?v=W5FTbteJc-k 
Schule. Anders als Gasser gewährt KainundAbel auch gewisse Einblicke in sein Privatleben, allerdings mit klar erkennbaren Grenzen. Nervenkrisen, Weinattacken vor der Kamera, die bei einigen Booktubern Nähe und Authentizität suggerieren sollen, gibt es bei ihm nicht. Aber beispielsweise Meinungs-Beiträge etwa zur Diversitäts-Debatte oder Pläne für den Sommer. Ein Plus und markant ist die gutturale Stimme des Sprechers, der man gerne zuhört. Die Videos sind in der Regel „stationär“, d.h. der Sprecher sitzt am Tisch und bewegt sich nicht durch Räume. Wie bei den meisten sind hinter ihm Bücherregale zu sehen, schaffen das korrespondierende Ambiente zum Inhalt des Videos. Allerdings erzeugt es hier nicht den Einschüchterungs-Effekt wie bei Gasser.

Damit wären die beiden männlichen Beispiele vorgestellt - die im Gesamten der Szene wie bereits erwähnt eine deutliche Minderheit bilden, und das gilt für die deutschen Booktuber ebenso wie für die englischen oder amerikanischen. Ilke Sayan aus Frankfurt betreibt ihren Kanal BuchGeschichten seit 2015. Neben Neuerscheinungen der Gegenwartsliteratur findet man bei ihr Klassiker und Graphic Novels, Krimis und Fantasy sind hingegen mehr oder weniger bewusst ausgespart. In ihrer Selbstvorstellung im „Kanalinfo“ betont sie die Bedeutung eines „regen Austausches mit anderen Bücherliebhabern“35 - und sieht man sich die Kommentarfunktion ihrer jeweiligen Videos an, kann man erkennen, dass dieser Wunsch erfüllt wird. Kaum jemand unter den anderen deutschen Booktubern hat eine solche Kommunikationsdichte mit den Usern wie Ilke Sayan. BuchGeschichten verfügt über gegenwärtig 9030 Abonnenten, auch dies für deutsche Verhältnisse durchaus ein Spitzenplatz. Die Aufrufe ihrer Beiträge gehen alle in mehrere Tausende, das Video "Jahresfavoriten 2020“36 wurde über 9000x angesehen, übertroffen nur von „Bücherregal vorher und nachher“"37 mit fast 15.000 - einem Format, das ja nicht nur bei Booktubern beliebt ist, sondern bei Bibliophilen aller Couleur: Wie ordne ich eine Bibliothek, einen privaten Buchbestand? Ihre Lese-Vlogs, worin Buchbesprechungen sich mit einigen Außenaufnahmen und Bildsequenzen der lesenden, kochenden, pflanzenden Protagonistin abwechseln, gehören zu den besten dieser Form. Themenvideos origineller Art wie „Düstere und schaurige Bücher“38, „Bücher zum Abtauchen“39 oder „Bücher mit unerwartetem Ende“ 40 sind eine Besonderheit, zu der dann auch eine „Klassikerwoche“ gehört, im Dezember 2020 beispielsweise 8 Texte von Charles Dickens. ${ }^{41}$ Ein Spezifikum bei BuchGeschichten ist die Rubrik „BuchGeschichten unterwegs“, wo u.a. von der Frankfurter Buchmesse, aus einem Literaturzug oder von einer Literatour durch London berichtet wird, auch über einen Tag als Gast im Suhrkamp-Verlag. Ilke Sayan sucht den Kontakt zu Institutionen der Buchwelt wie Verlagen, Buchhandlungen, Messen, diversen Veranstaltungsforen rund ums Buch. Darüber

35 https://www.youtube.com/c/BuchGeschichten/about

36 https://www.youtube.com/watch?v=MjND1Uj7ges

37 https://www.youtube.com/watch?v=fNA_XCah0rw

38 https://www.youtube.com/watch?v=MjfHQI4Ob3o

39 https://www.youtube.com/watch?v=2q2iTXWn0t0

40 https://www.youtube.com/watch?v=2_xeqs5P8LA

41 https://www.youtube.com/watch?v=2L4LAvlYfjM 
hinaus lässt sie in gelegentlichen Vlogs auch Einblicke in die Entstehung ihrer Videos zu. Ihre Beiträge vermitteln durch eine ruhige, nie überdrehte Präsentation Gediegenheit, Ernsthaftigkeit, ohne je langweilig oder belehrend zu wirken. Ilke Sayan erfüllt das Image einer guten Freundin, mit der man alles über Literatur besprechen kann, was einem so einfällt. Ihre Bemerkungen und Erläuterungen sind immer sachlich, Vorlieben oder Abneigungen gut begründet, wobei die Subjektivität des Urteils selbstreflexiv betont wird. Ich würde ihren Kanal als den besten unter jenen bezeichnen, die nicht wie Gasser oder KainundAbel schon eher im Lager der Professionals stehen. Eine rundum sympathische, sachkundige, urteilsfähige Booktuberin, die auch Adressaten erreicht, die Bücher nicht „berufsbedingt“ lesen - und deshalb für den Einsatz etwa im gymnasialen Unterricht im Bereich Literatur bestens geeignet ist.

Leoni von Leonieoverthere ist die Jüngste unter den ausgewählten Beispielen und zeigt, dass die Boooktuber-Szene bis in eine Generation reicht, die von Anfang an digital aufgewachsen ist. Leonie hat ihren Kanal 2014, als sie 20 Jahre alt war, eröffnet und gehört mit 591 Abonnenten aktuell zu den kleineren Kanälen. Dafür ist sie aber sehr aktiv, platziert bis zu 3 Videos mit durchschnittlich 15 Minuten pro Woche. Schon in ihrem Kanalinfo weist sie darauf hin, dass sie vielseitig interessiert sei und deshalb neben Büchern auch „Videos zu meinem persönlichen Leben, Hobbys und meiner zweiten großen Leidenschaft: der Auseinandersetzung mit der Geschichte ${ }^{\text {“42 }}$ postet. Dass sie mit allem „ein wenig unterhalten“ will, wird offen gesagt - aber diejenigen Beiträge, die sich auf Literatur begrenzen, sind von beeindruckender Kompetenz und Selbständigkeit des Urteils. Eines ihrer jüngsten Videos (19.3.2021) bringt eine Rezension zu Max Frischs Montauk und bietet eine ebenso kluge wie vorurteilsfreie Würdigung eines Buches, das ja ein Alterswerk ist und vermutlich bei vielen weiblichen, auch älteren Booktuberinnen als „sexistisch“ klassifiziert werden könnte. Leonie gesteht zwar ein, dass manche Aspekte des Buches ihr fremd bleiben, arbeitet aber die Konflikte und Problematik einer autobiographischen Fiktion überzeugend heraus. Leonieoverthere lässt ihre Zuschauer darüber hinaus am Studenten-Alltag teilhaben, der aktuell wie bei den meisten Studenten in Europa im Distanzunterricht verläuft, sie ist schon im zweiten Corona-Semester. Neben den typischen Booktube-spezifischen Sparten findet man eine Wohnungs-Präsentation $^{43}$ (Teil des Studenten-Alltags) oder auch einen Beitrag über „Meine ersten Rollschuhe“ ${ }^{44}$ Hoffentlich eine Ausnahme bleibt ein Video aus dem Februar 2021, „Meine skincare Routine 2021“.45 Leonie hat literarisch erkennbare Vorlieben, etwa für Max Frisch, moderne Klassik - und verzichtet auf das Meiste von dem, was erfolgreichere Booktuberinnen so vorstellen, nämlich Young Adult Literature oder Romantic-Reihen. Leonieoverthere scheint mir besonders für jüngere Studierende geeignet, die solide und doch empathische Ausführungen zu den von ihr besprochenen Büchern suchen und denen auch sie im Literaturstudium mit großer Wahrscheinlichkeit begegnen werden.

42 https://www.youtube.com/channel/UCso67IyGupBUeJHAsfcA8vg/about

43 https://www.youtube.com/watch?v=TnU6a_Tgzg

44 https://www.youtube.com/watch?v=oV063hH5opY

45

https://www.youtube.com/watch?v=V5mygKXwsws 
Als letztes Beispiel aus dem deutschsprachigen Bereich möchte ich den Kanal von Sophie Palme aus Berlin mit dem Titel VerStand vorstellen. VerStand ist seit 2006 dabei, ihre Betreiberin ist Ende 20 und Skandinavistik-Studentin, was sich in der Auswahl ihrer Bücher niederschlägt. Ihr Interessengebiet ist aber weiter, umfasst etwa neben russischen, norwegischen und deutschsprachigen Klassikern auch Graphisches und Mangas, Fantasy und Science-Fiction. Dass sich Booktuber unabhängig zu halten versuchen, dies gewissermaßen als Ehrencodex verstehen, zeigt sich an einer Äußerung in ihrem Kanalinfo: „Ich bitte darum, mir KEINE Rezensions- oder Leseexemplare zuzusenden“. ${ }^{46}$ Mit 4550 Abonnenten gehört sie in die Gruppe der Erfolgreicheren, ihre Aufrufe liegen in der Regel im vierstelligen Bereich. Die Videos von VerStand erscheinen durchschnittlich einmal im Monat und beschränken sich strikt auf Bücher, in den bekannten Formaten wie Lesemonat, Lieblingsbücher, Lese-Vlog, Lesevorhaben, Klassiker oder Neuzugänge. Persönliches, Privates gibt es hier weder filmisch - sieht man vom gleichbleibenden Setting, ihrem schmalen Arbeitszimmer, ab - und auch die bei vielen Booktubern präsentierten Bookish-Facts gehen bei ihr nicht über Mitteilungen zu Lesegewohnheiten hinaus. VerStand macht seinem Namen insofern Ehre, als die Beiträge immer sachorientiert sind, d.h. auf jede Art Ablenkung durch Ambiente, Kleidung, Außenwelt etc. verzichtet. Sophie Palme ist eloquenter als die meisten anderen deutschen Booktuber, vergleichbar etwa mit KainundAbel. Bei ihr paart sich Leidenschaft mit Sachlichkeit, was eine gute Synthese ergibt, nicht zuletzt bei einer Form, die es bei Booktubern nicht häufig gibt: dem Verriss. Desinteresse, Distanz, Nicht-Gefallen ja - aber bleibt dies bei vielen anderen eher „gefühlig“, argumentativ kaum abgestützt - ist dies anders bei VerStand: Knausgards Debütroman Aus der Welt etwa oder die Neuübersetzung von Dostojewskis Ein grüner Junge (bzw. früher Der Jüngling) kritisiert beredt und auch hier leidenschaftlich, was ihr an diesen Büchern misslungen scheint. Sophie Palme ist feministisch engagiert, was aus diversen Äußerungen herauszuhören ist, aber auch in ihrer kontinuierlichen Präsentation von aktuellen Schriftstellerinnen ebenso wie „vergessenen“, z.B. Erika Manns Werk. VerStand versammelt allein schon durch ihre lange Präsenz eine umfangreiche, vielleicht die umfangreichste Sammlung profilierter Videos zur Literatur und ihrer Geschichte, ist dabei weitestgehend auf Klassiker ausgerichtet und wäre deshalb eine ideale digitale Literaturgeschichte live, die Studierenden des Faches gute Dienste leisten könnte.

Emmie ist erst seit 2018 bei Booktube, gehört aber mit 2.745.000 Aufrufen ihrer Videos gewissermaßen in eine andere Liga, vergleicht man sie mit den deutschen Beispielen. Fast 65.000 Abonnenten sind ebenfalls eine Reichweite, die für den deutschsprachigen Raum wohl kaum zu erreichen sein dürfte - es sei denn, man weicht den Charakter des Formats auf, wie bereits angedeutet. Der Kanal emmie ist in Kanada ansässig und ihre Eignerin stellt sich im Kanalinfo vor „as an english lit and classical studies student“, „review all the many books I meet, and try to create cozy, calming content“ ${ }^{47}$ Die Frequenz ihrer Videos ist hoch, häufig gibt es mehrere pro Woche. Beeindruckend ist die Mannigfaltigkeit der Seite, gerade in Hinsicht auf all das, was sich zum Thema Buch machen

46 https://www.youtube.com/c/VERStand/about

47 https://www.youtube.com/c/emmie00/about 
lässt: Neben den bekannten Sparten präsentiert emmie gewissermaßen Einführungskurse in die Lektüre klassischer Literatur, „tips for beginners“, ${ }^{48}$ die so gut gemacht sind, dass sie wirklich geeignet sind, Barrieren abzubauen. „Come thrifting for books with me“49 schildert ausgiebige Buchhandlungsbesuche, am beeindruckendsten aber ist die Gründung des „Dark Academics Book Club“50, in dem sie im Livestream mit vier anderen bekannten Booktuberinnen gemeinsam ausgewählte Bücher bespricht. Dieses Format ist markant länger als Vlogs, das Beispiel von Anfang März, „Furies and Julius Caesar Live Show ${ }^{\prime 51}$, dauerte eine Stunde und zwanzig Minuten. Das Konzept zitiert einerseits den Film „Club der toten Dichter“, andererseits das Musik-Label „Dark Academia Music“, das ebenfalls bei youtube zu finden ist. Dieser Live-Performance folgten immerhin beinahe 6.000 Zuseher. Die Kommentarfunktion wird bei emmie intensiv genutzt - Äußerungen wie „oh, to have a group of friends to discuss books with like this“ zeigen, dass ein Bedürfnis nach literarischem „Salon“ solch digitaler Originalität durchaus vorhanden ist. Der Erfolg von emmie in so kurzer Zeit erklärt sich durch ihre sympathische Persönlichkeit, einer Art Girl Next Door, der es tatsächlich gelingt, eine „cozy“-Atmosphäre herzustellen - und dem unglaublichen Einfallsreichtum mit dem sie das Thema „Buch“ in immer neuen, nie aber sachfremden Kontexten erscheinen lässt. Etwa in „Dickens vs Tolstoy: The Great Debate with CarolynMarieReads“, einer anderen sehr gefragten Booktuberin aus Kanada, wo in einem ebenso schlagfertigen wie anregenden Disput über die beiden Romanciers diskutiert wird. Emmie führt ganz offensichtlich ihr Publikum auch gerne ein Stück weit in ihr privates Leben ein, allerdings ohne jeden Exhibitionismus, wie er ansonsten gerade in der anglo-amerikanischen Community durchaus vorkommt. Spaziergänge, Wohnungsbesichtigungen - gelegentlich auch offene Darstellung von Krisen mit der Bitte um Ratschläge und Meinungen. Dies insgesamt ergibt die Konstellation eines freundlich-freundschaftlichen Kreises, der über das zentrale Thema Literatur auch zu anderer Nähe zusammenfindet.

Die angloamerikanische Version von Booktube, wesentlich offensiver, auch showaffiner, repräsentiert sehr gut Emma Angeline, die schon 2013 im Alter von 14 Jahren begonnen hat, einen Book Channel zu betreiben. Die Autorin, die hinter diesem Label steht, stammt aus Belgien, lebt und studiert aber in England, sämtliche ihrer Videos sind in Englisch. Ihr impulsiver, gelegentlich zur Theatralik neigender Stil ist äußerst konträr zum deutschen, aber auch zu dem von emmie, kommt aber gut an. 48.500 Abonnenten und 3.776.000 Aufrufe mit Einzelaufrufen bis zu 40.000 sind bemerkenswerte Zahlen. Auch sie bietet Live-Auftritte, die sich aber eher selten mit Büchern beschäftigen, häufiger mit durchaus intimen Themen wie „Bi visibility and internalised misogyny and the feminist agenda" ${ }^{2}$ oder „emma being enthusiastic for 105 mins“ .53 Man kann davon

48 https://www.youtube.com/watch?v=RqRLC4vnJEc

49 https://www.youtube.com/watch?v=uDSxXLnw4kI

50 https://www.youtube.com/watch?v=2DFsoLh2Mlk

51 https://www.youtube.com/watch?v=MunGW8mG_7s

52 https://www.youtube.com/watch?v=R-71PSFjayY

53 https://www.youtube.com/watch?v=wSOlwmWh8MA 
ausgehen, dass ihre Attraktivität, vitale, lebhafte Ausstrahlung, ihre feminine Erscheinung eine beträchtliche Anzahl männlicher Abonnenten anzieht, die nicht unbedingt primär an den Büchern interessiert sind. Die Frau hinter Emma Angeline studiert Film und Comparative Literature in London, beides ist in ihren Videos erkennbar. Sie sind technisch hochprofessionell gestaltet und die vorgestellten Bücher aus allen möglichen Genres und Kulturen. Eindeutig ist, dass sie sich als Entertainerin versteht, bei der Leidenschaft, Empathie, die Performance über ausgewogenen Urteilen steht. Auch in den „reinen“ Buchbeiträgen ist sie meist rasch bei Erlebnissen, persönlichen Geschichten, die nicht immer etwas mit dem besprochenen Buch zu tun haben. Die Vermittlung zum Buch, zum Lesen gelingt hier über die Person, die „Sache“ tritt eher dahinter zurück. Bemerkenswert ist bei ihr auch die Offenheit in Sachen Beziehungen, Sexualität, sie thematisiert Gender-Fragen ohne sich als rigide Feministin zu sehen. Bisexualität und Schmink-Videos passen bei Emma Angeline durchaus zusammen, ihr Charme und ihre Lebhaftigkeit lassen es nie ideologisch oder missionarisch werden. Ihr Kanal scheint mir für die Entwicklung des Formats Booktube zu stehen: Ohne solche „Entertainer“ werden sich U- und E-Booktuber immer weiter auseinanderentwickeln. Was zu bedauern wäre, weil am wirksamsten für digitale Leser-Gewinnung eine gute Mischung von beidem wäre, sozusagen die Synthese aus VerStand und Emma Angeline.

Die Schwächen eines Formats wie Booktube sind evident, scheinen mir aber eher in der Breite als bei den ausgewählten Beispielen anhängig, sind also nicht konditional, nicht „systembedingt“. Eine gewisse Oberflächlichkeit, was kritische Kompetenz und sprachliche Ausdrucksfähigkeit angeht, eine gelegentlich beliebig anmutende Auswahl der Bücher sind bei vielen Booktube-Auftritten durchaus festzustellen. Ergo: ein eher unwissenschaftlicher Umgang mit Literatur. Auch die häufig starke Fixierung auf die Person der Vermittlung, deren Lebenszusammenhänge anstatt der Sache, ist zu beobachten. Der Gestus scheint nicht selten dem einer „Werbung“, eines „Advertising“ zu entsprechen, wozu die immer häufiger zu beobachtende Koppelung an Life-StyleThemen passt. Ein gewisser Narzissmus mancher Booktuber ist offensichtlich. Nur - gilt nicht das meiste davon auch für Vertreter der „klassischen“ Literaturkritik? Gab es keine Fixierung auf die Person, keinen Narzissmus etwa bei Marcel Reich Ranicki, Joachim Kaiser, Hans Mayer - oder heute bei Thea Dorn oder Denis Scheck? Hat das Bündnis mit Entertainment nicht schon viel früher Einzug gehalten in die Literaturkritik - und nicht erst im digitalen Zeitalter? Man lese Kurt Tucholskys Rezensionen und Glossen zum literarischen Leben der Zwanziger Jahre. ${ }^{54}$ Dass Literaturkritik immer auch kommerzielle Aspekte hatte, wird nicht einmal der bereitwilligste Idealist bestreiten. Es gibt also Differenzen, mag sein gelegentlich gravierende. Dies aber abgewogen gegenüber den Vorteilen von Booktube scheinen mir diese zu überwiegen. Nur einige davon seien abschließend noch einmal angeführt.

Booktuber erreichen vor allem ein jüngeres, aber durchaus auch generationsübergreifendes Publikum. Lebendigkeit, Vielfalt und ein offenes Bekenntnis zum UnterhaltungsAspekt des Formats machen Buchvorstellungen zu einem Erlebnis multimedialer, auch

54 Tucholsky, Kurt: Literaturkritik. Mit einer Vorbemerkung von Fritz. J. Raddatz, Reinbek b. Hamburg, Rowohlt, 1972. 
mehrdimensionaler Art. Die Sprache bei Booktube, entfernt vom „Fachchinesisch“, erreicht Menschen, die ansonsten am Gespräch über Literatur nicht teilnehmen würden - und dieses „Gespräch“, die literarische Kommunikation, findet in Form von Kommentaren oder Vernetzungen auch nachweislich statt. Die oft beobachtete Einsamkeit des Lesers wird zumindest partiell aufgehoben, Selbstbewusstsein gestärkt, mitgeteilt. Die Offenheit dieser Diskurse darf man durchaus als ein Stück Demokratisierung im Literaturbetrieb verstehen, die eigentlich geforderte Interaktivität im System Sender/ Empfänger ist nicht nur technisch, sondern gewissermaßen auch psychologisch möglich gemacht. Last but not least: Die Bookchannels bieten jedem potentiellen Leser etwas, man muss nur „seinen“ entsprechenden Kanal finden. Die Hauptsache ist, man bringt Lust zum Lesen mit - und ist dies nicht eine Basis-Forderung für so gut wie alles, was mit Literatur zu tun hat, auch die Literaturwissenschaft? Deshalb glaube ich, dass das Phänomen Booktube auf jeden Fall in einem ausgewählten Bereich seinen Platz im Rahmen der Literaturwissenschaft finden sollte. Dafür ein praktisches didaktisches Modell vorzulegen wäre Thema eines anderen, anschließenden Beitrags. Nur als Anregung: Die Erstellung eines Booktube-Videos als Partner- oder Gruppenarbeit im Rahmen des Literaturunterrichts würde gleich mehrere Kompetenzen initiieren: aktive sprachliche Auseinandersetzung mit einem Thema, einen eigenen Stil zu entwickeln, vom Konsumenten zum Produzenten werden und Verantwortung für eine Präsentation übernehmen.

Technisches Know How und intellektuelle Fähigkeit, Können und Verstehen würden so als mögliche Einheit ein eigenständiges Format hervorbringen.

Fazit: Es lohnt sich hier, eine „Schöne Neue Welt“ des Umgangs mit Literatur zu betreten, Booktuber läuten nicht das Sterbeglöckchen der Literaturkritik, ganz im Gegenteil!

\section{Literaturverzeichnis}

Albrecht, Wolfgang (2001): Literaturkritik. Stuttgart/Weimar: Verlag J.B. Metzler.

Eagleton, Terry (2016): Literatur lesen. Eine Einladung, Stuttgart: Reclam.

Ernaux, Annie (2020): Trennen, Verbinden. In: (Hrsg.): Raabe, Katharina und Wegner, Frank:

Warum lesen. Mindestens 24 Gründe. Frankfurt/M.: Suhrkamp Verlag, S. 79-88.

Kittler, Friedrich (1985): Aufschreibesysteme 1800/1900. München: Wilhelm Fink.

Reckwitz, Andreas (2020): Kleine Genealogie des Lesens. In: (Hrsg.): Raabe, Katharina und Wegner, Frank: Warum lesen. Mindestens 24 Gründe. Frankfurt/M.: Suhrkamp Verlag, S. 31-45.

Schiller, Friedrich (1991): Wallensteins Tod. III/2. In: (Hrsg.): Fricke, Gerhard und Göpfert, Herbert G.: Friedrich Schiller. Sämtliche Werke. Zweiter Band. Dramen II, München: Hanser Verlag.

Singer, Wolf (2020): Immaterielle Realitäten. In: (Hrsg.): Raabe, Katharina und Wegner, Frank: Warum lesen. Mindestens 24 Gründe. Frankfurt/M.: Suhrkamp Verlag, S. 148-163.

Tucholsky, Kurt (1972): Literaturkritik. Mit einer Vorbemerkung von Fritz. J. Raddatz, Reinbek b. Hamburg, Rowohlt.

Walther, Klaus (2004): „Haben Sie das alles gelesen?“ In: Ders. Bücher sammeln, München: dtv, S. 17-23. 


\section{Internetlinks}

Alle links wurden am 10.4.2021 noch einmal zur Kontrolle aufgerufen http:/ / buzzaldrins.de/2014/02/20/sigrid-loffler-im-gesprach/

https://www.youtube.com/watch?v=4Y11ZQsyuSQ

https:/ / www.sueddeutsche.de/politik/nach-fall-rezo-ziemiak-will-newsroom-einfuehren-und-ar-

beitsablaeufe-in-cdu-zentrale-aendern-1.4622154

https://www.dw.com/en/how-booktubers-are-changing-book-marketing/a-18633901

https://www.youtube.com/watch?v=q7JFbXlekZA

https://www.youtube.com/watch?v=LT46gQvzP2A

https://www.youtube.com/c/LITERATURISTALLES/about

https://www.youtube.com/watch?v=qcAugg_kHY0

https://www.youtube.com/watch?v=9Zqg0glOhHI

https://www.youtube.com/user/KainUndAbel2/about

https://www.youtube.com/watch?v=8eca-Es9Dkk

https:/ / www.youtube.com/watch?v=omHRyX2ErOg

https://www.youtube.com/watch?v=W5FTbteJc-k

https://www.youtube.com/c/BuchGeschichten/about

https://www.youtube.com/watch?v=MjND1Uj7ges

https://www.youtube.com/watch?v=fNA_XCah0rw

https://www.youtube.com/watch?v=MjfHQI4Ob3o

https:/ / www.youtube.com/watch?v=2q2iTXWn0t0

https://www.youtube.com/watch?v=2_xeqs5P8LA

https://www.youtube.com/watch?v=2L4LAvlYfjM

https://www.youtube.com/channel/UCso67IyGupBUeJHAsfcA8vg/about

https://www.youtube.com/watch?v=TnU6a__Tgzg

https://www.youtube.com/watch?v $=$ oV063hH5opY

https://www.youtube.com/watch?v=V5mygKXwsws

https://www.youtube.com/c/VERStand/about

https://www.youtube.com/c/emmie00/about

https://www.youtube.com/watch?v=RqRLC4vnJEc

https://www.youtube.com/watch?v=uDSxXLnw4kI

https://www.youtube.com/watch?v=2DFsoLh2Mlk

https://www.youtube.com/watch?v=MunGW8mG_7s

https://www.youtube.com/watch?v=R-71PSFjayY

https://www.youtube.com/watch?v=wSOlwmWh8MA

\section{Dr. Habil. Jürgen Eder / eder@ff.jcu.cz}

Jihočeská univerzita v Českých Budějovicích, Filozofická fakulta, Ústav česko-německých areálových studií a germanistiky

Branišovská 31a, 37005 České Budějovice, CZ

This work can be used in accordance with the Creative Commons BY-SA 4.0 International license terms and conditions (https://creativecommons.org/licenses/by-sa/4.0/legalcode). This does not apply to works or elements (such as image or photographs) that are used in the work under a contractual license or exception or limitation to relevant rights 
